\title{
Ciudadanos, defensa, libertad: análisis de contenido informatizado de la Constitución Española (1978)
}

\section{Citizens, defense, freedom: analysis of computerized content of the Spanish Constitution (1978)}

\author{
Dr. Daniel Barredo lbáñez \\ Escuela Superior Politécnica de Chimborazo (Riobamba, Ecuador) \\ danielbarredo@aol.com \\ Dr. Martín Oller Alonso \\ Escuela Superior Politécnica de Chimborazo (Riobamba, Ecuador) \\ martin.olleralonso@gmail.com \\ Dr. José María Pérez Zúñiga \\ Universidad de Guayaquil (Guayaquil, Ecuador) \\ imp-z@telefonica.net \\ Recibido: 20 de marzo de 2013 \\ Aceptado y Publicado: 30 de julio de 2013
}

\begin{abstract}
Resumen
La Constitución Española de 1978 definió las reglas de juego de la democracia actual, porque es el instrumento jurídico que regula las relaciones entre gobernantes y gobernados, y que representa la unidad de las autoridades, los procedimientos y sus contenidos. A través del análisis de contenido informatizado evaluamos en este artículo los recursos lingüísticos empleados en el documento legislativo más importante de España. Es este un procedimiento novedoso hasta ahora, mediante el cual pretendemos examinar las diferentes narrativas que integran el significado global del texto. En conjunto hemos analizado las 40 palabras-tema más frecuentes del texto, su posicionamiento léxico y sus relaciones jerárquicas. El análisis terminológico de la CE ilustra, en primer lugar, una abundancia de referencias a los tipos de
\end{abstract}


normas y al ordenamiento jurídico en sí y, en segundo lugar, a las propias instituciones y a los derechos y libertades de los ciudadanos.

\begin{abstract}
The Spanish Constitution of 1978 defined the rules of democracy today, as it is the legal instrument that regulates the relations between rulers and ruled, and represents the unity of the authorities, procedures and contents. Through computerized content analysis in this paper we evaluate the linguistic resources used in the most important work of legislation in Spain. This is a new technique, by which we intend to examine the various narratives that make up the overall meaning of the text. Altogether we analyzed the 40 topic - words most frequent words of the text, its lexical positioning and their hierarchical relationships.

On the one hand, the analysis of the vocabulary of the Spanish Constitution illustrates an abundance of references to the types of standards and the legal system itself and, on the second hand, many allusions to the institutions themselves and the citizen rights and freedoms.
\end{abstract}

Palabras Clave: Constitución Española de 1978; España; legislación; derecho; análisis de contenido informatizado.

Key Words: Spanish Constitution of 1978, Spain; legislation; law; computerized content analysis.

\title{
1. Introducción
}

Es común hablar de las constituciones como aquellas normas que obligan a todos los sujetos de la comunidad política, empezando por los poderes públicos. Se trata, por tanto, de la norma más importante del ordenamiento jurídico, pues se la da la comunidad política a sí misma para organizarse a través de las instituciones que aplicarán las normas con los límites y los procedimientos establecidos en la propia constitución. Obviamente, la Constitución Española de 1978 (en adelante, CE) responde a ese planteamiento, y más allá de las influencias derivadas de la historia de España y de un momento histórico concreto (la ley fundamental de Bonn de 1949, la Constitución Italiana de 1947 y la Constitución Republicana Española de 1931), la constitución es un instrumento jurídico que obliga por igual a gobernantes y a gobernados, pues representa una unidad de autoridades, procedimientos y contenidos. 
Esta realidad se pone de manifiesto en este artículo, si bien desde una perspectiva de estudio bien distinta a la de la doctrina constitucionalista. Con una propuesta metodológica novedosa, el análisis terminológico de la CE ilustra las preocupaciones del constituyente en el sentido antedicho, pues abundan los términos referidos en primer lugar a los tipos de normas y al ordenamiento jurídico en sí, a las propias instituciones en segundo lugar y a los derechos y libertades de los ciudadanos; es decir, lo que ha venido a identificarse con la parte orgánica y la parte dogmática del texto constitucional.

En un momento en que el llamado "problema regional" (las aspiraciones de autogobierno de las nacionalidades históricas y la organización territorial) vuelve a discutirse en España, este estudio ilustra la riqueza y flexibilidad de la norma fundamental del ordenamiento jurídico y del propio bloque de la constitucionalidad para adaptarse al desarrollo social y a la evolución de una comunidad que aspira a vivir democráticamente.

\section{Metodología}

En este artículo hemos empleado como metodología principal el análisis de contenido informatizado, una técnica que permite evaluar ingentes cantidades de material textual de forma precisa y objetiva (Popping, 2000; Chen, Evans, Battleson, et al., 2011). El software elegido ha sido CatPac ${ }^{1}$, un programa que es capaz de organizar los términos de un texto determinado en una red neuronal artificial. En dicha red, cada palabra - clave (llamada en este trabajo palabra - tema), constituye una neurona o punto de activación. Al introducir un algoritmo de búsqueda, en este caso de \pm 1 colocación, CatPac enseña las relaciones jerárquicas que se establecen en función de:

a) Las frecuencias de aparición.

b) El posicionamiento léxico (mediante la instrucción dada en el algoritmo).

El procedimiento ha sido definido y explicado ya en algunos trabajos previos (Barredo, 2012; Barredo y Oller, 2012a, 2012b), si bien en las páginas que mostraremos a continuación -

\footnotetext{
${ }^{1}$ Category Package, programa desarrollado por el Dr. Joseph Woelfel, de la Universidad de Buffalo (Nueva York). Para más información sobre el creador o sobre la empresa distribuidora, se aconseja visitar la web oficial: http://www.galileoco.com/N_catpac.asp.
} 
antes de abordar el apartado de los resultados-, extractaremos y explicaremos los pasos previos instrumentales, los cuales sintetizamos mediante el gráfico siguiente:

\section{Figura 1. Pasos instrumentales del análisis y síntesis de su descripción}

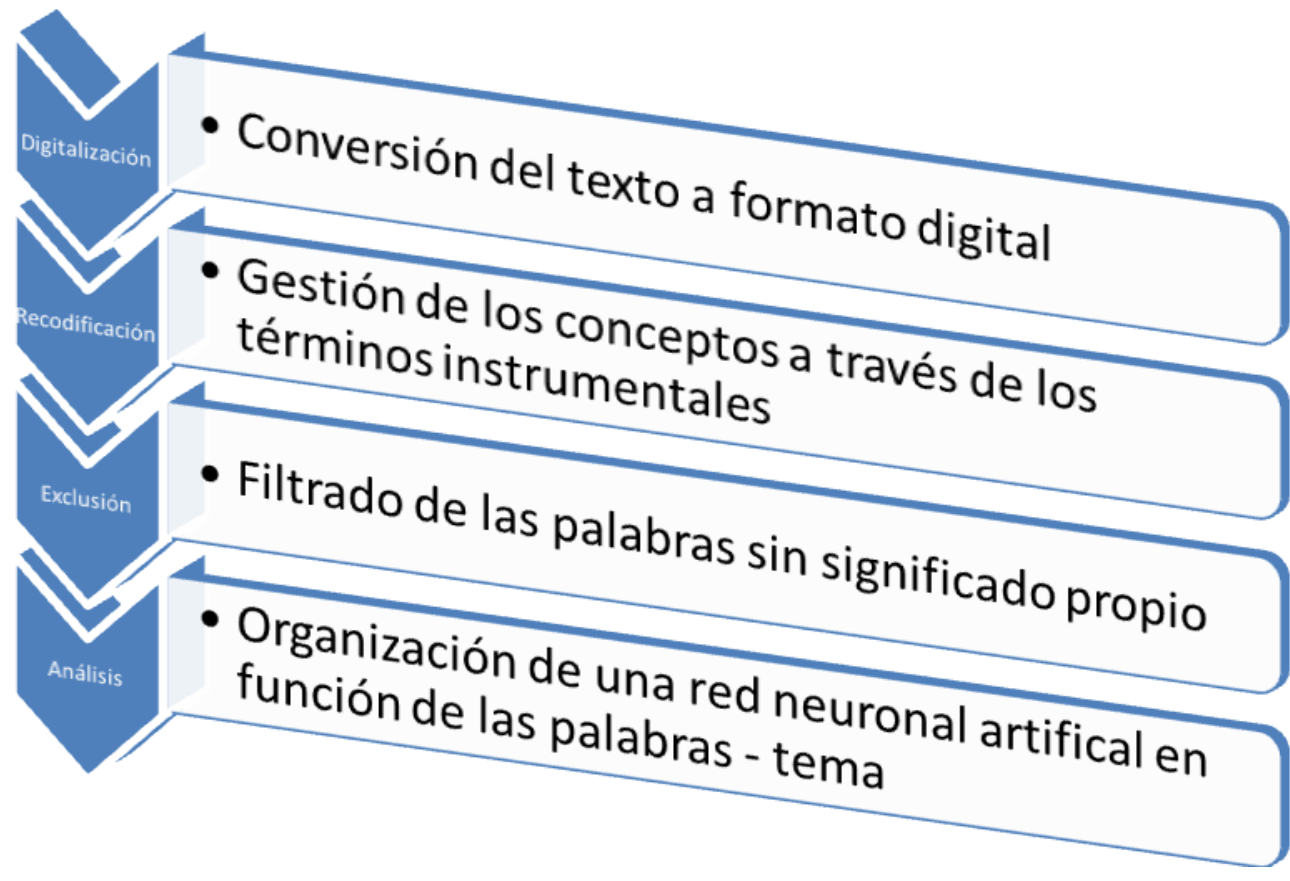

\section{Fuente: Elaboración propia}

\section{Primer paso: digitalización textual}

El primero de los pasos, antes de realizar un análisis de contenido informatizado, exige convertir un texto en bloques textuales digitalizados; en este caso descargamos la CE desde la web oficial del Congreso español ${ }^{2}$.

\section{Remodificaciones}

\footnotetext{
${ }^{2}$ Disponible en:

http://www.congreso.es/portal/page/portal/Congreso/Congreso/Hist_Normas/ConstEsp1812_1978/Const1978 (consultado el 24/01/2013).
} 
Una vez descargado el texto jurídico y convertido en un fichero de texto, realizamos una observación preliminar, tras de la cual apreciamos una ingente cantidad de material léxico específico. El programa utilizado solo contabiliza los términos unimembres, por lo que hemos diseñado unos términos instrumentales (son aquellos términos creados artificialmente para gestionar un análisis de contenido informatizado); en esos términos instrumentales hemos incluido las variantes terminológicas tanto plurales como singulares. También se han tenido en cuenta las omisiones de parte de la cadena, como por ejemplo cuando hemos hallado en el texto la palabra Tribunal, que ha sido agregada -en su caso- al término instrumental TRIBUCONSTITUCIONAL, el cual como se aprecia en su raíz designa al Tribunal Constitucional. Los términos instrumentales, como se verá en la siguiente tabla, se han recodificado en letras mayúsculas para facilitar su localización durante la fase analítica.

\begin{tabular}{|l|l|}
\hline \multicolumn{2}{|l|}{ FIG. 2. Recodificación: términos o conceptos textuales e instrumentales } \\
\hline Término o concepto textual & Término instrumental \\
\hline Administración autónoma & ADMINISTRACIÓNAUTÓNOMA \\
\hline Administración civil & ADMINISTRACIÓNCIVIL \\
\hline Administración de Justicia & ADMINISTRACIÓNJUSTICIA \\
\hline Administración del Estado & ADMINISTRACIÓNESTADO \\
\hline Administración Local & ADMINISTRACIÓNLOCAL \\
\hline Administración militar & ADMINISTRACIÓNMILITAR \\
\hline Administración pública & ADMINISTRACIÓNPÚBLICA \\
\hline Comunidades Autónomas & COMAUTONOMAS \\
\hline Congreso de los Diputados & CONGDIPUTADOS \\
\hline Consejo de Estado & CONESTADO \\
\hline Consejo General del Poder Judicial & CONGEPOJUDICIAL \\
\hline Consejo de Gobierno & CONGOBIERNO \\
\hline Consejo de Ministros & CONMINISTROS \\
\hline Cortes Generales & CORTGENERALES \\
\hline De acuerdo & DEACUERDO \\
\hline Decretos-leyes & DECRETOSLEYES \\
\hline Delegación legislativa & DELELEGISLATIVA \\
\hline Estatutos de Autonomía & ESTAAUTONOMIA \\
\hline Interés general & INTERESGENERAL \\
\hline Iniciativa legislativa & INILEGISLATIVA \\
\hline Leyes de bases & LEYESDEBASES \\
\hline
\end{tabular}




\begin{tabular}{|l|l|}
\hline Mayoría absoluta & MAYOABSOLUTA \\
\hline Mayoría de edad & MAYORÍADEEDAD \\
\hline Mayoría simple & MAYOSIMPLE \\
\hline Poder Judicial & POJUDICIAL \\
\hline Poderes constitucionales & PODERESCONSTITU \\
\hline Poderes públicos & PODERESPUBLICOS \\
\hline Presupuestos Generales del Estado & PRESUGENEESTADO \\
\hline Proposición de ley & PROPOSICIÓNDELEY \\
\hline Proyecto de Estatuto de Autonomía & PROYECTOESTAAUTO \\
\hline Proyecto de ley & PROYECTODELEY \\
\hline Tribunal Constitucional & TRIBUCONSTITUCIONAL \\
\hline Tribunal de Cuentas & TRIBUCUENTAS \\
\hline Tribunales de Honor & TRIBUHONOR \\
\hline Tribunal Supremo & TRIBUSUPREMO \\
\hline Seguridad Social & SEGUSOCIAL \\
\hline Leyes orgánicas & LEYESORGANICAS \\
\hline Fuente: Elaboración propia &
\end{tabular}

El desarrollo de estos términos instrumentales permite coleccionar esos términos compuestos presentes en un texto, pero también permite filtrar determinados términos indeseables (como la locución de acuerdo), que mencionaremos en el próximo paso instrumental.

\section{Exclusiones}

Los proyectos basados en el análisis de contenido informatizado suelen establecer algunas exclusiones (Tian y Stewart, 2005), con el objetivo de centrar la evaluación terminológica no en las palabras, sino en concreto en las palabras - tema (son los términos, como hemos mencionado ya, que identifican estructuralmente los contenidos de un texto). Para obtener tales términos clave, hemos filtrado:

- Las palabras carentes de significado distintivo. Las preposiciones, los artículos determinados 0 indeterminados, los demostrativos, los pronombres personales, los verbos más frecuentes del castellano (ser, estar, parecer, haber, tener, poder, deber, ir), los adverbios, las conjunciones, las locuciones, etc. 
- Algunos términos que solo se activan en función del contexto, como por ejemplo los sustantivos años, casos, términos, apartado, artículo, ejercicio, miembros, régimen, plazo (porque introducen sensaciones significativas muy difuminadas), o bien esos otros que tienden a confundirse con elementos de una locución o de un verbo, como forma, generales, así como la locución en general.

\section{Palabras analizadas}

Después de estos procesos preliminares, necesarios para centrar el análisis y evitar injerencias léxicas indeseadas, se han registrado algunas variaciones con respecto del texto original:

\begin{tabular}{|c|c|c|c|}
\hline Palabras & Caracteres sin espacios & Palabras procesadas & $\begin{array}{l}\text { Caracteres } \\
\text { espacios }\end{array}$ \\
\hline 18.698 & 100.631 & 18.066 & 99.170 \\
\hline
\end{tabular}

Si atendemos a la figura 2, observamos que se ha producido una disminución del 3,38\% ( $n=632$ palabras) del material terminológico inicial, dado el elevado número de recodificaciones. Anotamos estas variaciones porque el objetivo de un análisis de contenido informatizado, como subraya Popping (2000), es transparentar su gestión por si algún otro investigador desea validar los resultados a partir de los mismos pasos instrumentales.

\section{Resultados}

Para comprender mejor las palabras - tema que integran la siguiente tabla, tal y como hemos desarrollado ya en otros proyectos (Barredo, 2012; Barredo y Oller, 2012a, 2012b), hemos añadido unas marcas cromáticas que rotulan su pertenencia a un campo semántico distintivo:

- Verde: jurídico. Es un vocabulario fuertemente connotado por su naturaleza jurídica.

- Naranja: institucional. Son términos que describen instituciones, nociones institucionales u órganos del Estado. 
- Azul: neutro. Son nociones que se activan en función del contexto, por lo que poseen un carácter esencialmente neutro.

- Rojo: principios. Alude a nociones vinculadas con derechos y libertades.

\begin{tabular}{|l|l|l|}
\hline \multicolumn{3}{|l|}{ FIG. 4. Cuarenta palabras - tema de la Constitución española (1978) } \\
\hline Palabra - tema & Frecuencia & $\%$ \\
\hline LEY & 125 & 8,6 \\
\hline DERECHO & 91 & 6,3 \\
\hline GOBIERNO & 91 & 6,3 \\
\hline $\begin{array}{l}\text { COMUNIDADES } \\
\text { AUTÓNOMAS }\end{array}$ & 87 & 6,0 \\
\hline ESTADO & 80 & 5,5 \\
\hline CONSTITUCIÓN & 59 & 4,1 \\
\hline DERECHOS & 57 & 3,9 \\
\hline CÁMARAS & 51 & 3,5 \\
\hline REY & 49 & 3,4 \\
\hline CORTES GENERALES & 46 & 3,2 \\
\hline LEYES ORGÁNICAS & 38 & 2,6 \\
\hline CONGRESO & 37 & 2,5 \\
\hline FUNCIONES & 36 & 2,5 \\
\hline PRESIDENTE & 36 & 2,5 \\
\hline ESTATUTO & 32 & 2,2 \\
\hline AUTONOMÍA & & \\
\hline PODERES PÚBLICOS & 31 & 2,1 \\
\hline LEYES & 30 & 2,1 \\
\hline REGULARÁ & 28 & 1,9 \\
\hline ESPAÑA & 26 & 1,8 \\
\hline TRIBUNAL & 25 & 1,7 \\
\hline CONSTITUCIONAL & & 1,7 \\
\hline ESPAÑOLES & 24 & 1,6 \\
\hline LIBERTAD & 23 & \\
\hline & & \\
\hline
\end{tabular}




\begin{tabular}{|c|c|c|}
\hline TRATADOS & 22 & 1,5 \\
\hline INICIATIVA & 21 & 1,4 \\
\hline PERJUICIO & 21 & 1,4 \\
\hline PRINCIPIOS & 21 & 1,4 \\
\hline SENADO & 21 & 1,4 \\
\hline COMPETENCIAS & 20 & 1,4 \\
\hline MAYORÍA ABSOLUTA & 20 & 1,4 \\
\hline NORMAS & 20 & 1,4 \\
\hline PÚBLICO & 20 & 1,4 \\
\hline CIUDADANOS & 19 & 1,3 \\
\hline LEGISLACIÓN & 19 & 1,3 \\
\hline LIBERTADES & 19 & 1,3 \\
\hline TERRITORIO & 19 & 1,3 \\
\hline DEFENSA & 18 & 1,2 \\
\hline ESTABLEZCA & 18 & 1,2 \\
\hline JUDICIAL & 18 & 1,2 \\
\hline MAYORÍA & 18 & 1,2 \\
\hline PROCEDIMIENTO & 18 & 1,2 \\
\hline
\end{tabular}

Fuente: Catpac / Elaboración propia

Enlistamos, a continuación, las palabras - tema que hemos discriminado en cada uno de los grupos ${ }^{3}$ :

a) Jurídico. Es este el grupo más numeroso, con el $40 \%(n=16)$ de las palabras - tema más frecuentes: Ley, derecho, Constitución, derechos, leyes orgánicas, Estatuto de Autonomía, leyes, regulará, tratados, perjuicio, competencias, mayoría absoluta, normas, legislación, judicial, mayoría.

b) Institucional. La corriente institucional, que identifica a los órganos o a los representantes del Estado, aglutina al 27,5\% $(n=11)$ del léxico significativo más repetido: Gobierno, Comunidades Autónomas, Estado, Cámaras, Rey, Cortes Generales, Congreso, presidente, poderes públicos, Tribunal Constitucional, Senado.

\footnotetext{
${ }^{3}$ Esta clasificación solo tiene un valor orientativo. El recuento de los términos, de carácter cuantitativo, tiene que complementarse con un análisis interpretativo porque los ordenadores, como nos recuerda Diefenbach (2001), no pueden realizar por sí solos las inferencias.
} 
c) Neutro. La elevada precisión exigida al análisis (a través de las recodificaciones y de las exclusiones), hacía predecible una escasa contabilización de palabras - tema caracterizadas por su valencia neutra. En total solo el 17,5\% $(n=7)$ de los 40 términos más frecuentes pueden ser atribuidos a este campo: Funciones, iniciativa, España, españoles, establezca, territorio, procedimiento.

d) Derechos y libertades. En cuarto lugar se sitúa, con un $15 \%(n=6)$ de los términos más representativos, ese campo que menciona realidades que remiten a los principios básicos: Libertad, principios, público, ciudadanos, libertades, defensa.

Según los grupos anteriores, observamos en el texto constitucional un desnivel hacia las dos primeras esferas de sentido:

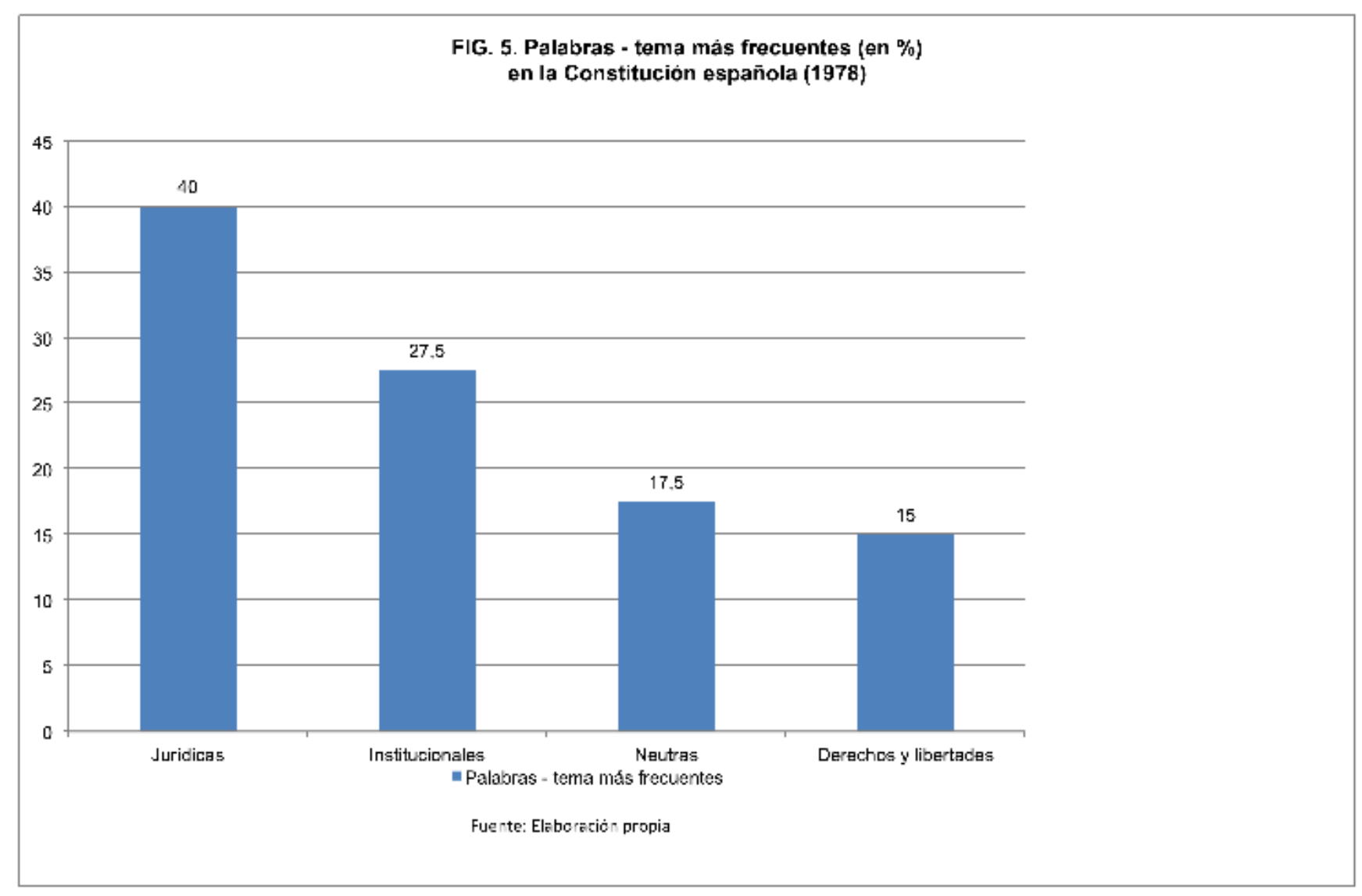


Pero además de anotar las principales corrientes léxicas que estructuran al texto constitucional, el otro punto de interés de este trabajo es visualizar las relaciones que se establecen entre esos términos. Cada uno de los círculos que contiene el siguiente mapa identifica la posición de una palabra - tema; la cercanía entre unos términos y otros refleja, asimismo, la tendencia a aparecer en los mismos contextos:

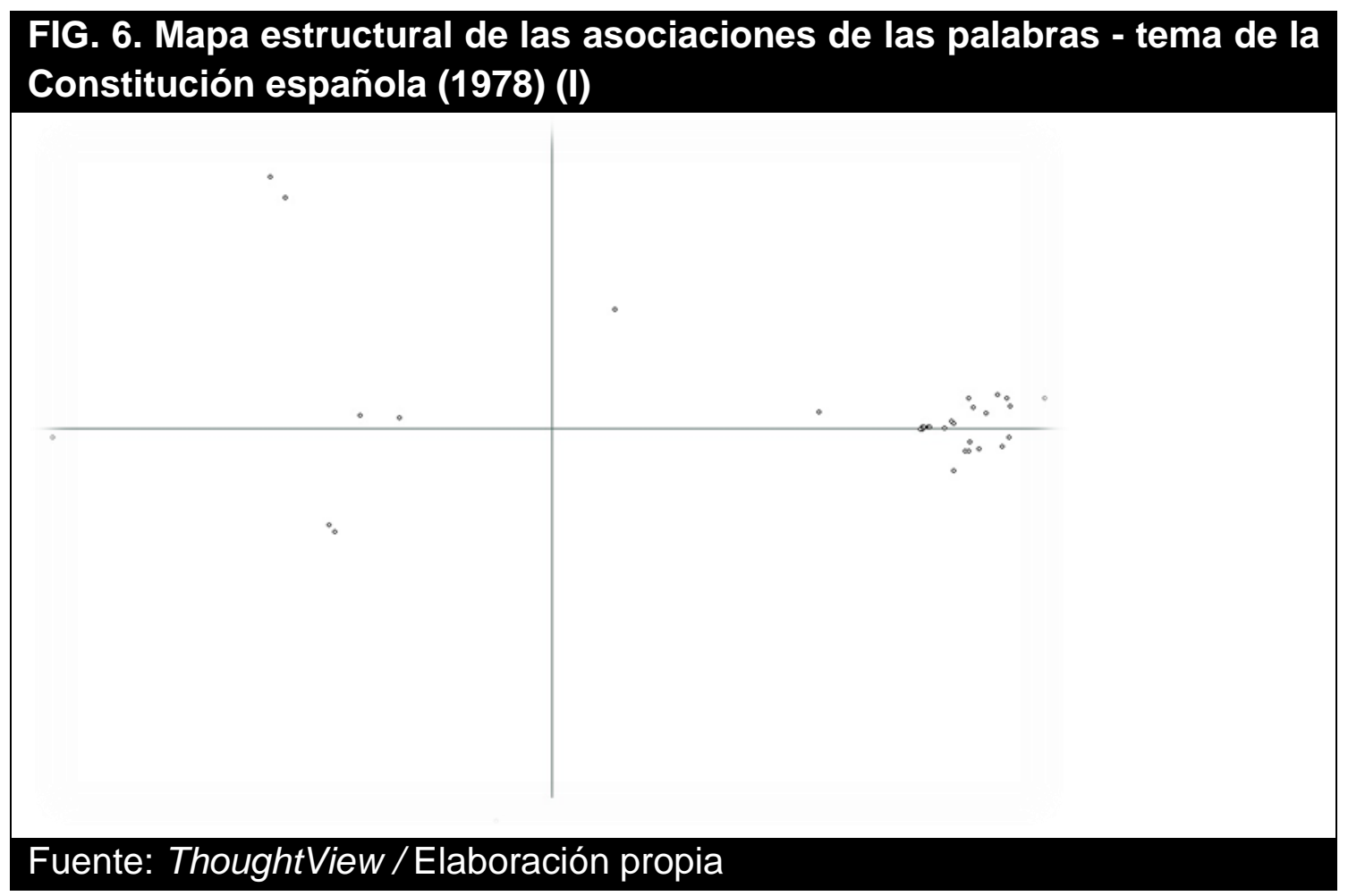

La imagen anterior muestra, por un lado, una dispersión elevada entre las palabras - tema que configuran el vocabulario más repetido de la Constitución, salvo en el grupo que se aprecia en la parte derecha, el cual concentra la mayor parte del caudal léxico. En la captura siguiente, agregamos las etiquetas identificativas (o términos instrumentales) para mejorar la apreciación de esa macrotendencia: 


\section{FIG. 7. Mapa estructural de las asociaciones de las palabras - tema de la Constitución española (1978) (II)}

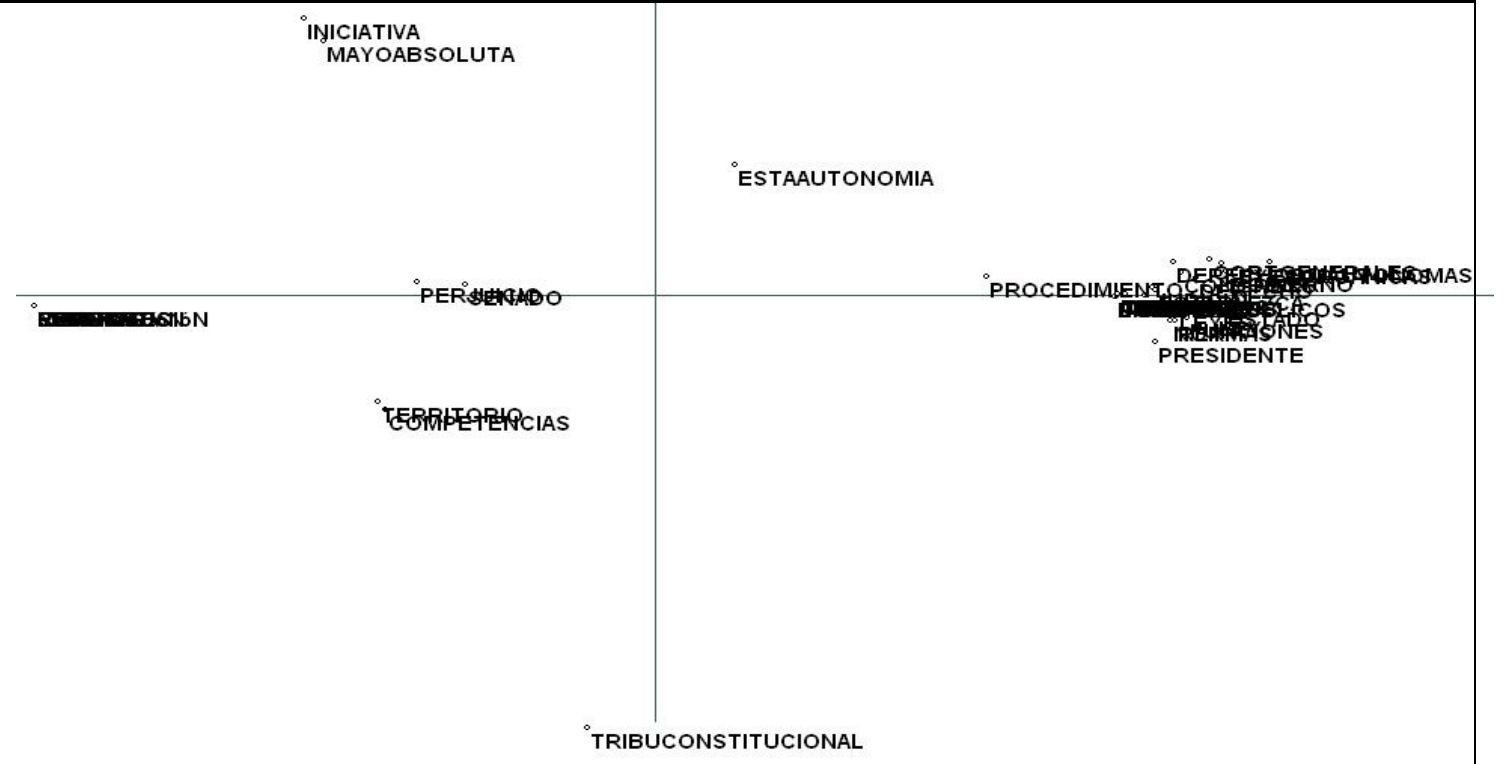

Fuente: ThoughtView / Elaboración propia

Dentro del bloque disperso hallamos sugerentes asociaciones, reveladoras de importantes aspectos del texto constitucional, como las que se establecen entre iniciativa y mayoría absoluta, o bien entre perjuicio y Senado o territorio y competencias ${ }^{4}$. El grupo sincopado que aparece en la parte izquierda del mapa contiene una curiosa definición del discurso analizado; al conectar los siguientes términos prácticamente obtenemos una respuesta coherente sobre el significado de este texto: "Constitución, Cámaras, España, público, regulará, españoles, legislación, mayoría”.

Pero la corriente más interesante está formada por ese conjunto de la parte derecha, el cual hemos ampliado para observar más detenidamente las complejas relaciones que se establecen entre sus palabras - tema. En total 23 de los términos temáticos oscilan en esta esfera:

\footnotetext{
${ }^{4}$ La lista de las asociaciones léxicas mencionadas aparece recogida en el anexo 1 a este trabajo; en el anexo ofrecemos el dendrograma, cuyas relaciones son más estables y exactas que las que aparecen en el mapa perceptual, que es una herramienta que solamente se emplea para facilitar la visualización.
} 


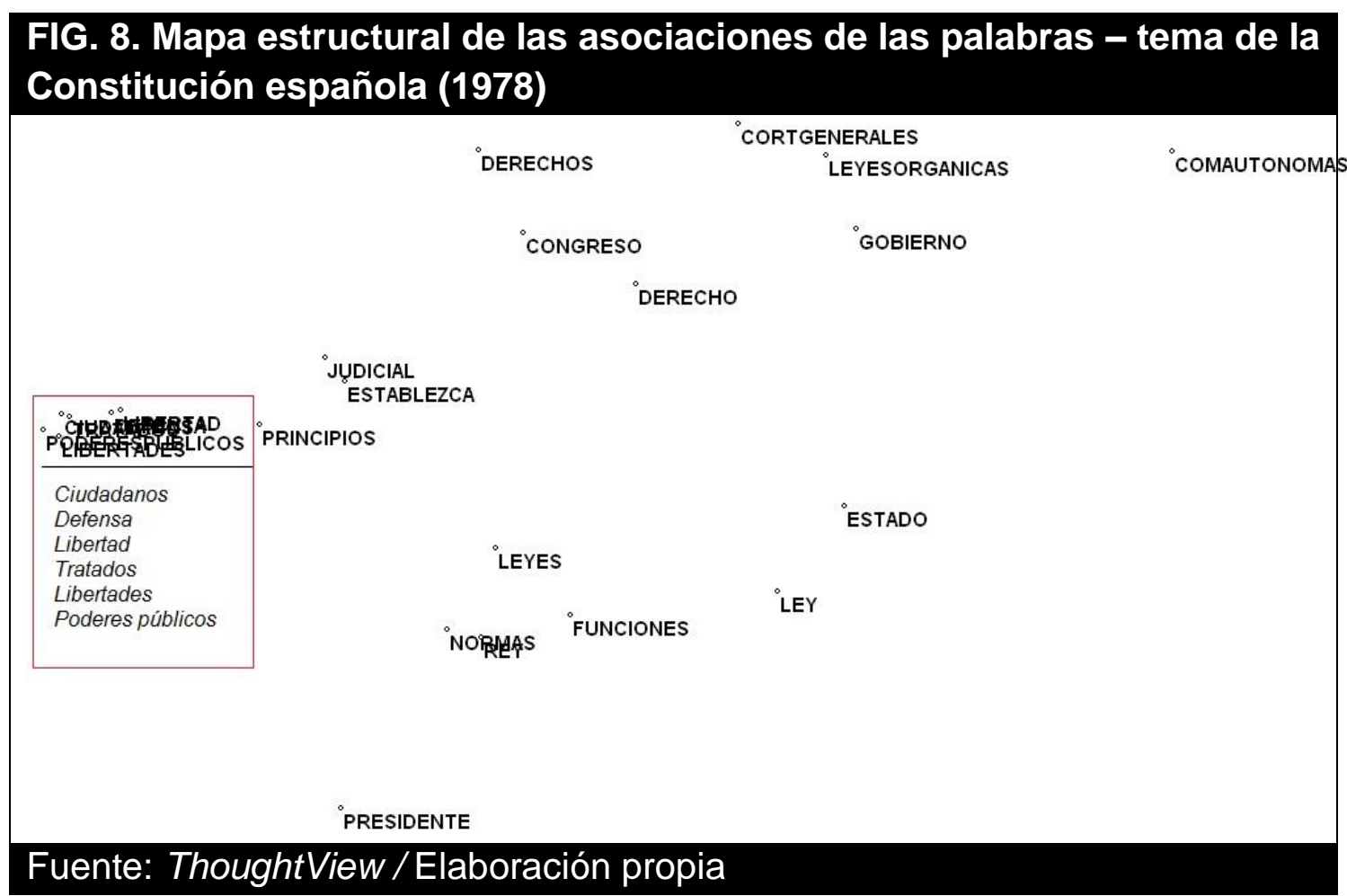

Pero si en el bloque de asociaciones dispersas obteníamos una respuesta asociativa de carácter más formal (la Constitución como reguladora de la legislación de la mayoría de los españoles), en esta esfera, y en niveles de separación contextual elevados, hallamos una significativa acumulación de términos relacionados con un término tan significativo como principios: Ciudadanos, defensa, libertad, tratados ${ }^{5}$, libertades, poderes públicos ${ }^{6}$.

En el mapa anterior orbitan asociaciones que rubrican determinadas realidades, como por ejemplo la importancia del Congreso como gestor de la convivencia (al vincularse a derecho y a derechos); el estrecho vínculo que existe entre las Cortes Generales y las leyes orgánicas; o la relación que se establece entre el presidente -y sobre todo, el rey-, con respecto de las normas, de la ley, y de las leyes, entre otros.

\footnotetext{
${ }^{5}$ Los tratados son convenios internacionales, contratos entre países o instituciones públicas, de obligado cumplimiento.

${ }^{6}$ Hemos encuadrado este bloque y explicado mediante cursivas los términos que tienden a sincoparse.
} 


\section{Conclusiones}

Entre los principales hallazgos de nuestro análisis, resulta muy llamativo el hecho de que las palabras - tema neutras hayan alcanzado un porcentaje similar al de esos términos que abanderan derechos y libertades. En contra de lo que podía predecirse, los términos más representativos del texto constitucional español están caracterizados por sus vinculaciones a nociones jurídicas o institucionales.

La actitud centralista de la Constitución aparece enumerada en las constantes alusiones a palabras - tema como la mayoría o la mayoría absoluta; dentro de ese posicionamiento como carta de convivencia, destaca también la importancia concedida a las autonomías, y a los representantes públicos estatales encarnados por el rey y el presidente del Gobierno.

Pero quizá la dimensión más sugerente que define a este texto colectivo es su ensalzamiento de la libertad, su defensa de las libertades, una conceptualización que -tal y como hemos visto- corona simbólicamente las asociaciones léxicas de los términos más frecuentes. Son esos derechos y libertades los que configuran la parte dogmática de la Constitución, exigibles ante los tribunales, y directamente en amparo ante el Tribunal Constitucional, tomados de la Declaración Universal de los Derechos Humanos. Ese es el corazón de esta Constitución aunque late -según la tabla aportada en la figura 3-, a bajas frecuencias. A altas frecuencias hallamos sobre todo la presencia más abultada de un discurso trabado por alusiones jurídico - institucionales, probablemente a causa de la urgencia del momento de su redacción. No olvidemos que la Constitución suele explicarse como un ejemplo de acuerdo entre las élites (Sánchez - Cuenca y Aguilar, 2009), acuerdo que impulsó la adopción de un lenguaje funcional, tendente más a lograr pactos y a profundizar sobre todo en aspectos relativos a la distribución del poder entre los territorios.

En este trabajo hemos descrito, mediante una técnica tan novedosa como el análisis de contenido informatizado, los sentidos generales que atraviesan la Constitución de 1978. Es este, con todo, un estudio preliminar que principia un análisis comparativo sobre la evolución de los temas en las Constituciones históricas, un proyecto que nos permitirá medir las asociaciones léxicas que han conformado el imaginario del patrimonio constitucional español. 


\section{Bibliografía}

Barredo, D. (2012). El tabú de la expresividad real. Análisis del tratamiento informativo del rey Juan Carlos I en ABC.es y ELPAÍS.com (2009 - 2011) <tesis doctoral>. Málaga: Universidad de Málaga.

Barredo, D. y Oller, M. (2012a). "Tratamiento de género en las informaciones ofrecidas sobre el Día Internacional de la Mujer Trabajadora (2001 - 2010) en ABC.es", en Libro de Actas del I Congreso Internacional de Comunicación y Género (Coord., Suárez, J. C., Liberia, I. y Zurbano, B.). Sevilla: Editorial Mad.

Barredo, D. y Oller, M. (2012b). Género, ideología y prensa digital. La construcción informativa del Día Internacional de la Mujer Trabajadora en www.abc.es y www.elpais.com (2001 - 2010). Alicante: Revista Mediterránea de Comunicación.

Chen, H., Evans, C., Battleson, B., Zubrow, E., Woelfel, J. (2011). "Procedures for the precise analysis of massive textual datasets" en Communication \& Science Journal, 2011 Oct 10.

Diefenbach, D. L. (2001). "Historical foundations of computer-assisted content analysis", en Theory, method, and practice in computer content analysis (Ed., West, M. D.). Westport, CT: Ablex Publishing.

Popping, R. (2000). Computer-assisted text analysis. Londres: Sage.

Sánchez Cuenca, I. y Aguilar, P. (2009). "Terrorist Violence and Popular Mobilization: The Case of the Spanish Transition to Democracy", Politics \& Society, 37(3), España, junio, pp. 428-453.

Tian, Y. y Stewart, C. M. (2005). "Framing the SARS Crisis: A Computer-Assisted Text Analysis of CNN and BBC Online News Reports of SARS", Asian Journal of Communication, 15(3), Inglaterra, febrero, pp. 289-301. 


\section{ANEXO 1.-}

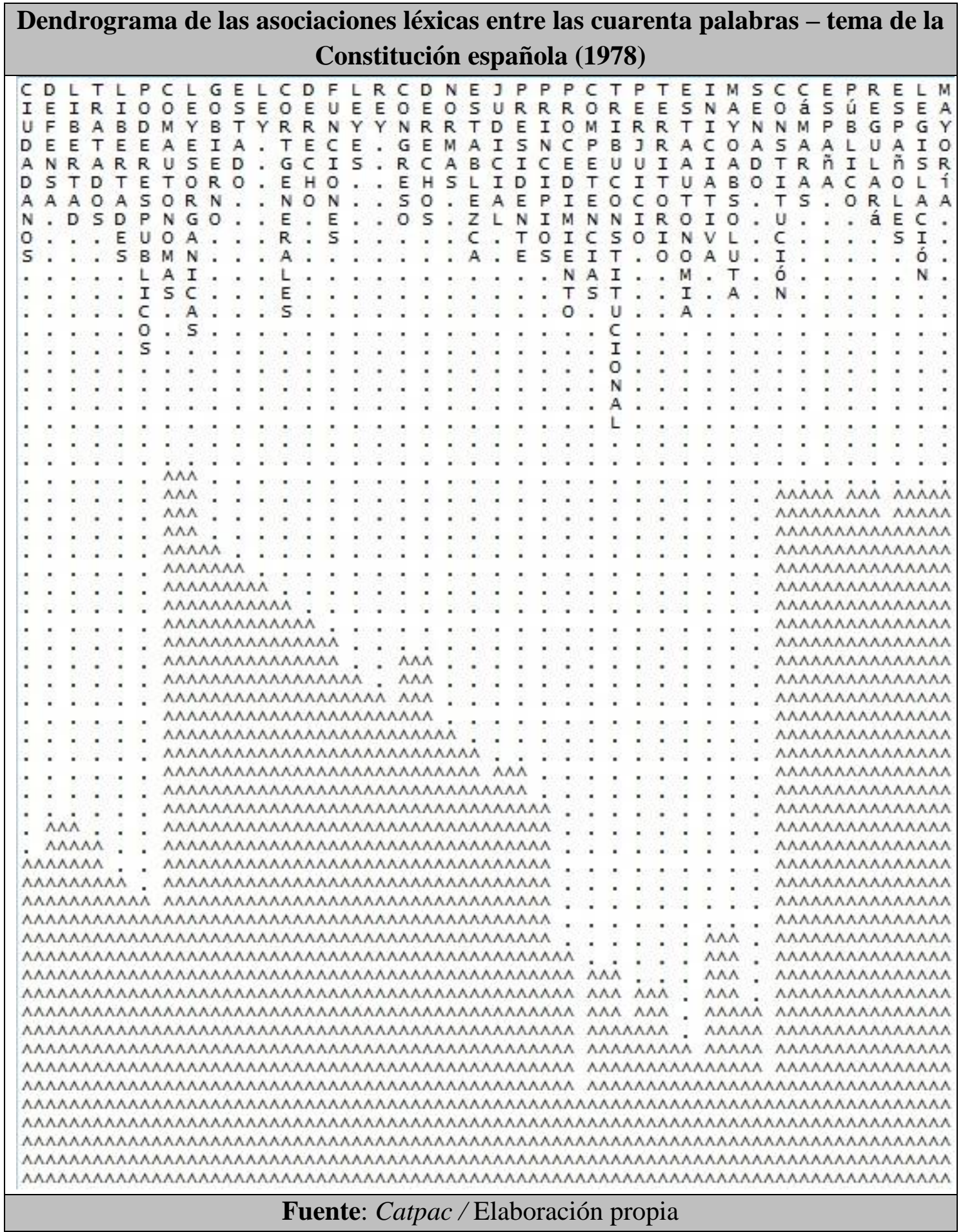




\section{Forma de citar este artículo en bibliografías}

Barredo Ibáñez, D.; Oller Alonso, M.; Pérez Zúñiga, J. M. (2013): "Ciudadanos, defensa, libertad: análisis de contenido informatizado de la Constitución Española (1978)", en Revista PANGEA, 4, páginas 93 a 109. Red Académica Iberoamericana de Comunicación. Recuperado el de de 2 de: http://www.revistapangea.org 